Summary.

I. Tuberculosis of the knee-joint is in the very large majority of cases initially a synovial infection, and remains so for a long period, and is sometimes entirely confined to the synovia.

2. X-ray examination is of no real value in the early diagnosis which must be made and treatment started long before X-ray changes are apparent.

3. Despite treatment the disease tends to involve the whole joint, leading to fibrous ankylosis and, in cases where nature is assisted by surgery, bony ankylosis.

4. Immobilisation must be combined with extension for a very long period, followed by ambulatory treatment in a weight-bearing caliper, with the sound foot raised on a patten; and later, gradual progress to weight bearing, the knee being protected from strain for many months.

5. There is usually a tendency to recurrence after periods of apparently complete quiescence.

\title{
THE TECHNIQUE OF EXCISION OF THE INTERNAL SEMILUNAR CARTILAGE
}

\author{
By K. I. NISSEN, F.R.C.S. \\ (Orth. Surg., E.M.S. Royal National Orthopaedic Hospital, Stanmore; \\ Hon. Orth. Surg. to the Harrow Hospital)
}

The differential diagnosis of internal derangements of the knee was described in detail by Bristow (1) in an excellent number of this Journal, copies of which are still available. I propose to describe a common technique for removal of the internal semilunar cartilage, and while doing so, to give the reasons for certain preferences. The surgeon aims to excise the cartilage as completely as possible with minimal trauma, and at the same time to inspect the joint. The operation must of course be performed under the strictest non-touch technique, the essential points of which have again been stressed by Fairbank

\section{Preparation for Operation.}

After a hot bath and scrub, the skin is prepared from the upper thigh down to and including the toes. The nurse or sister who prepares the patient should wear an efficient mask, for there is no doubt that some of the alarming infections which occur from time to time are respiratory in origin. I have known a streptococcal and a pneumococcal infection to develop in two successive patients from the same ward. Patients themselves tend to be talkative during preparation, and should lie down with heads turned away.

Three spirit preparations are given, iodine being avoided on account of some risk of dermatitis. The protecting towels need to be fixed securely either by a circular bandage or by strips tied at intervals. The whole of the leg from the groin downwards is included. The towels must not be disturbed in any way till the tourniquet has been applied and the patient is in position on the operating table, for otherwise the careful ward preparation may be rendered worthless.

\section{The Anaesthetic.}

Gas and oxygen is usually sufficient, as any venous congestion is of no account when a tourniquet is used. The deep sleep that follows induction by an intravenous anaesthetic should not be mistaken for surgical anaesthesia. If a spinal anaesthetic be given, the perineum is protected by a large pad of wool in case of sudden relaxation of the rectal sphincter.

\section{The Application of the Tourniquet.}

An Esmarch's bandage is entirely satisfactory, as the operating time is short and the femur well covered by muscle. Unless the assistant is skilled, the surgeon is wise to apply the nonsterile tourniquet himself. The limb, still encased in towels, is elevated and the rubber bandage is applied firmly, with successive turns just not overlapping, from the foot to the upper thigh. The next two or three turns completely overlap one another and are just firm. They act as a base which prevents the final constricting turns from cutting into the flesh. With a large patient two bandages may be required.

Entirely insufficient tension leads to venous congestion which can be recognised from the colour of the skin immediately the towels are removed. In such a case the tourniquet is released, and after the limb has been held vertical for a minute or two, it is quickly reapplied. On the other hand the tension may gradually become insufficient from diminution in bulk of 
the muscle under pressure, when persistent oozing develops at some stage during the operation. The tourniquet is at once released and if necessary applied again.

A record of the times of application and final release is a safeguard against leaving the tourniquet in position after the operation. Early and severe post-operative pain is usually due to constriction either by a forgotten tourniquet or by too firm bandaging. On no account should morphia be prescribed without inspection of the whole limb.

\section{The Position on the Operating Table.}

The patient's knee is placed exactly over the hinge for letting down the end of the table. A tubular sandbag three inches in diameter is placed transversely behind the lower hamstrings and prevents the limb from rolling. The towels are next removed from the foot and the assistant holds the leg up by gripping the great toe in a large swab. The end of the table is lowered and four sterile towels are applied. One large abdominal towel is tucked well up behind the thigh and covers the sound leg while another is spread over the patient almost down to the knee. A small towel secured with a sterile circular bandage is used to enclose the lower leg and foot, and comes high up behind the knee so that the surgeon may pull the tibia forwards on the femur without touching skin. Another small towel is clipped round the thigh just above the knee and leaves only a small area to be covered later by the skin towels.

The surgeon sits on a stool and the height of the table is adjusted so that the foot can be held between his knees. The operating light points downwards and inwards over his shoulder towards the periphery of the cartilage rather than the centre of the joint.

\section{The Skin Incision.}

The surface marking for the incision is obtained by pressing the points of a pair of skin forceps into the hollow over the joint line just medial to the patellar tendon (Fig. I). The



FIG. I.-The bony outlines of the right knee flexed to a right angle are shown, with the relationship of the incision to the points of skin forceps pressed into the joint space just medial to the patellar tendon. oblique incision, two and a half inches long, runs downwards and outwards at an angle of $30^{\circ}$ to the vertical, and ends just below and medial to the points of the forceps.

The advantages of this incision are several. The subcutaneous tissue is very loose and the short incision can be retracted to give more exposure than might be expected. When deepened, it provides good access to the front and to both sides of the medial tibio-fcmoral joint. Only terminal branches of nerves are severed and cutaneous anaesthesia is not complained of later. The vastus internus muscle is not exposed and no other structures are endangered. The wound is sutured with ease, and being in the line of muscle pull is not affected by a moderate degree of movement during convalescence. The shortness of the scar is a psychological aid to the return of normal function.

Of alternative incisions it may be fairly said that a patella-displacing incision is entirely unnecessary; that the short horizontal anterior incision gives very limited access; and that a long horizontal incision round the joint line introduces a risk of damage to the internal lateral ligament.

\section{Skin Exclusion.}

The skin knife and forceps are discarded and linen skin towels are applied over the edges of the wound: Most surgeons use ordinary towel forceps, but some find that extra large Michel clips secure the towels most effectively to the cut edge, are less traumatic, and can be used many times over. Complete skin exclusion is maintained throughout the operation, until only the skin remains to be sutured. 


\section{Deepening the Incision.}

The glistening quadriceps expansion, having been cleared of areolar tissue, is divided with a clean knife in the same direction as the skin incision. The fibres actually severed are mainly decussating ones. The edges are separated by narrow Langenbeck retractors and are cleaned for a short distance on the under side by light touches of the scalpel. This definition is well worth while, for it makes any confusion between layers when sewing up almost impossible. The extra-synovial fat is now divided, again in the same line. About the level of the joint space one or two transverse vessels are encountered. When the tourniquet has been particularly effective, the vessels may be difficult to recognise in continuity, but as they are cut across, small beads of dark blood stand out against the fat. If not ligatured at once, they are occluded with medium sized Kocher forceps which are allowed to hang down and do not interfere with the operation. These are the only vessels that require attention.

It now remains to enter the joint. The careful surgeon divides the synovial membrane at the upper end of the incision between a pair of Kocher forceps and so avoids any damage to the cartilage of the internal condyle. The incision is extended downwards, care being taken not to cut the anterior margin of the semilunar cartilage.

\section{Inspection of the Joint.}

Broader retractors of Paton's type are now gently inserted and an excellent exposure of the joint is obtained. The amount and character of the synovial fluid is noted. Minute fibres floating in clear synovial fluid are characteristic of a tear, but a blood-stained effusion suggests other damage. The articular cartilage is inspected with particular attention to the lateral side of the femoral condyle, which is the common site for osteochondritis dissecans. Synovial tags and fringes arising from the ligamentum mucosum and ligamenta S1. alaria are seldom of any importance and the temptation to excise them has to be resisted. The cruciate ligaments are identified in the intercondylar space. Though its posterior end now may be seen, the external cartilage is too remote for satisfactory examination.

\section{Inspection of the Internal Semilunar Cartilage.}

Most of the front half of the cartilage can be seen when the tibia is pulled strongly forwards, and damage in this region is easily recognised (Fig. 2a). A displaced "bucket handle" will be seen lying in the intercondylar space (Fig. $2 b$ ). If not displaced, it may be made to slip into the centre of the joint by pulling the tibia forwards. An early longitudinal tear without separation may be demonstrated by gently probing the cartilage opposite the internal lateral ligament (Fig. 2f). When inspection remains negative, the upper end of the tibia is pulled forwards to put the cartilage on the stretch. If no tags or flakes can yet be seen, a posterior tear may still be present in the hidden part of the cartilage. As the history and clinical examination have suggested a tear, the surgeon must proceed to excise the cartilage completely. To detach the anterior horn for direct traction and then, if no posterior tear is seen to excise only the front half of the cartilage, is not a procedure to be recommended.

Occasionally the surgeon may have found on inspection of the joint a lesion that gives symptoms like those of a damaged semilunar cartilage, such as an area of osteochondritis dissecans or a cartilaginous loose body. He then has to review the case and decide if the findings are sufficient to account for the symptoms and physical signs. If they are, the surgeon deals with the obvious lesion and leaves the cartilage alone.

\section{The Problem of Complete Removal of the Cartilage.}

Complete removal through an anterior incision depends upon operative skill and the strength of the cartilage in the region of the internal lateral ligament. If this part is damaged, either by the injury or by the surgeon's knife, it may rupture when traction is applied. The following cases illustrate the problems which constantly arise:-

\section{Torn Anterior Horn (Fig. 2a).}

A large proportion of undamaged peripheral cartilage remains and the cartilage should be completely excised without difficulty. 
'Bucket-handle' tear (Shown displaced in Fig. 2b).

About half of the cartilage remains attached and is strong enough to stand considerable strain. Complete excision is necessary, for the peripheral rim in the back of the joint may give trouble from secondary tears if not removed. The displaced "handle" may be removed as a preliminary, but the operator usually endeavours to remove the "handle" and the rim still united. If the rim tears across half way back, the remainder must be removed through a separate approach.

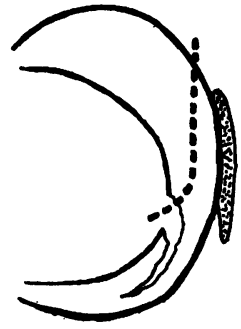

(a)



(d)

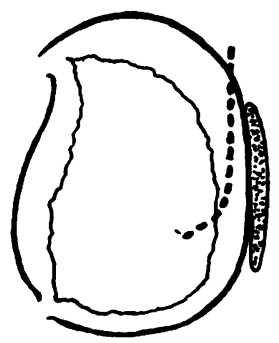

(b)

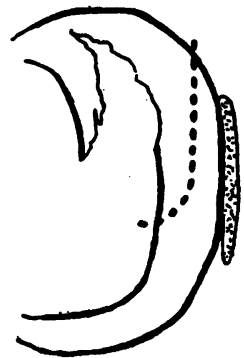

(e)



(c)

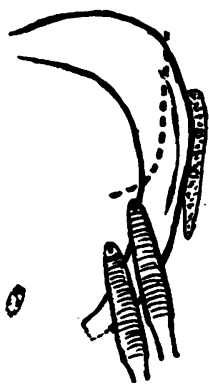

$(f)$

FIG. 2.-Diagrams showing typical lesions of the internal semilunar cartilage. The relation of the cartilage to the internal lateral ligament is shown. The dotted line represents the limit of direct vision. Fig. $2 f$ shows the anterior horn detached, with Ochsner forceps in position.
(a) Marginal tear of anterior horn.
(b) "Bucket-handle" tear shown displaced.
(d) Transverse tear.
(e) Tear of the posterior horn.
(c) Peripheral tear shown displaced.
(f) Early longitudinal tear without displacement.

Peripheral tear (Shown displaced in Fig. 2c).

Almost the whole cartilage lies in the intercondylar space and it is excised with ease. If the narrow rim is appreciable in amount, it is removed as far back as direct vision permits. Any posterior remainder will not give rise to further trouble.

Transverse tear of the middle of the cartilage (Fig. $2 d$ ).

Complete removal is attempted. If the cartilage tears across, the posterior half is removed through a separate incision. A transverse tear is not common but may be found in conjunction with a tear of the posterior horn of the external cartilage (Lambrinudi's double lesion) (3).

\section{Posterior horn tear (Fig. 2e).}

The whole cartilage can generally be removed. During excision it is most important to keep well out to the periphery of the cartilage right back to the limit of vision so as to minimise the risk of rupture. If this occurs, the remaining tag may be recovered in the intercondylar space, but a large and inaccessible remainder demands removal through a separate incision. 


\section{Freeing the Anterior Horn.}

The best type of knife to use is a narrow solid-bladed scalpel with a strong back. The detachable type of blade is dangerous, especially in the later stages of the operation. Blades of special design have been known to snap, and the recovery of a loose fragment from the posterior compartment of the joint may require a further incision and be extraordinarily difficult.

The front margin of the cartilage is freed by a vertical cut in the periphery just short of the soft capsular tissue. A blunt hook is passed round the cartilage and its point is made to appear in the cut. Upward tension is maintained and the incision is continued towards the transverse ligament and the anterior attachment, which is severed by an upward cut. The anterior quarter of the cartilage is now free and is held by one or two large Ochsner forceps firmly clamped obliquely across it (Fig. 2f). It is a simple matter to free the cartilage as far as the anterior margin of the internal lateral ligament, but special retraction is necessary opposite the ligament.

\section{Retraction of the Internal Lateral Ligament.}

A useful type of retractor is Paton's modification of Langenbeck's instrument. The straight blade is two inches long and half an inch wide and ends with a narrow lip. The blade is inserted between the ligament and the femoral condyle till the lip is felt to slip round the posterior margin of the ligament (Fig. 3a). Some instrument makers leave the lip too long

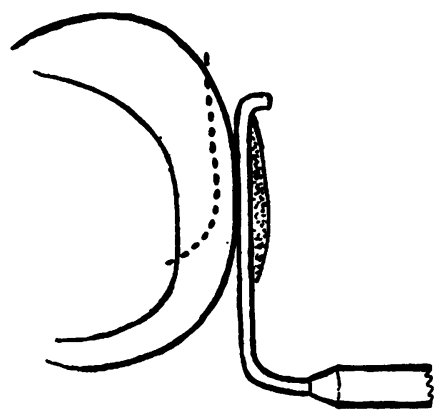

(a)



(b)

Fig. 3. -To show the relationship to the internal lateral ligament of Paton's modified Langenbeck retractor and Burrows' curved retractor.

and insertion is then difficult. This is serious, as both ligament and condyle are covered with synovial membrane which will react to any rough handling.

Burrows' instrument has a blade of similar dimensions but is curved to follow the periphery of the cartilage. As it has no lip it can be slipped into position with the greatest of ease. With either type of retractor it is impossible to damage the lateral ligament, provided the cut in the periphery of the cartilage is kept vertical.

\section{Freeing the Cartilage from the Ligament.}

Steady traction is kept up by the strong Ochsner forceps. The cartilage is said to be attached to the posterior two-thirds of the ligament. The attachment is by no means a fusion. Thus Brantigan and Voshell (4) even state that there is no strong fibrous attachment, and that a bursa is often interposed. It is freed under direct vision as far back as possible, keeping well out to the periphery. As soon as the incision is past the ligament, the cartilage is free to straighten out and tear away from the capsule up to the posterior attachment, but for the opposition of the tibio-femoral joint surfaces. To get the cartilage to slip through the joint the Ochsner forceps, still under tension, are carried laterally. Displacement is not difficult with a slack joint and a strong cartilage, but the estimation of the force that may be used with safety comes only with experience. The tension must be well under control in case the cartilage should displace suddenly and then rupture, leaving tags attached posteriorly. 


\section{Division of the Posterior Attachment.}

A two-inch Paton's retractor is used to give access to the intercondylar space. The posterior attachment is pulled forwards by traction on the cartilage or behind the upper end of the tibia. It is then divided under direct vision by a downward cut. Some prefer to hold the cartilage up and to divide the attachment from underneath, but flakes of tibial articular cartilage may be sliced off. After a final inspection for any remaining tags the joint is closed.

\section{Access to the Posterior Horn.}

In practice, skilled surgeons seldom find this necessary. The indications for a posterior incision have already been mentioned. No relaxation of full non-touch technique may be permitted, however annoying the circumstances.

The incision is made with the knee still at right angles. The sartorius muscle then lies below the level of the knee joint, and the long saphenous vein and nerve which follow its posterior border, are carried backwards. The joint space just posterior to the internal lateral ligament is defined and exposed through a vertical incision two inches in length. If the landmarks are obscure, as in a fat person, a dissector is introduced through the anterior incision and passed round the posterior border of the internal lateral ligament to act as a guide. The white rim of the cartilage is then easily defined and can be freed under direct vision right back to the posterior attachment.

\section{Closure of the Anterior Incision.}

The well defined aponeurotic layer is again held aside by small retractors leaving the surgeon free to suture the synovial membrane and extra-synovial fat. 20-day No. o catgut is used on a strong half-circle No. 3 Murphy's cervix needle. Thinner needles may snap. The stitch is a continuous one and either simple or mattress in type. This suture is of some

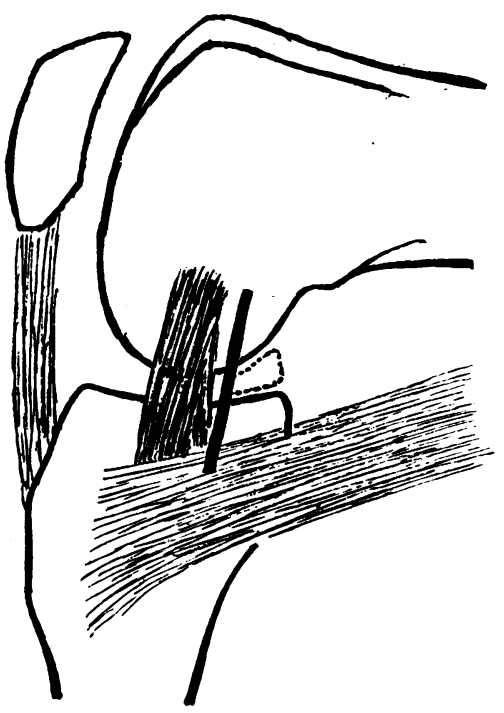

FIG. 4.-To show the relationship of the posterior incision to the internal lateral ligament. haemostatic value, and full bites are taken over the joint line where the extra-synovial fat contains the principal vessels. An assistant maintains tension with an angled catgut forceps, the smooth blades of which do not fray the gut. Comyns Berkeley's tissue forceps are invaluable for tying the knots, as their blades meet accurately.

The skin is next retracted and the knee is held almost straight. The edges of the incision in the quadriceps expansion come together and are easily sutured with the same type of needle and gut. The skin towels are now removed and the wound is closed with a continuous suture of medium silkworm gut on a straight skin needle.

\section{Application of Dressings.}

Over the usual gauze a layer of sterile cotton wool is fixed by a sterile circular bandage. Wool, which need not be sterile, is applied in two thick layers from the lower calf to mid-thigh, each layer being compressed by turns of broad circular bandage. The tourniquet is then released and the time noted.

The value of the thick woollen dressing is almost entirely as a comfortable splint limiting passive movement to $30^{\circ}$. Surgeons who claim that it limits effusion by pressure rather than by rest, apply the bandage very tightly and include the foot to avoid oedema; but the return of blood after removal of the tourniquet increases the tension still further and the dressing may have to be reapplied less tightly on account of pain. Others deny any value to the firm wool dressing and apply a minimal amount with little pressure; but a restless or a foolish patient may then bend the leg too freely and not maintain an essential degree of rest. 


\title{
Inspection of the Excised Cartilage.
}

The cartilage is orientated and sketched in the operation record. Old tears are easily recognised. Clean transverse nicks in the edge of the cartilage are almost certainly produced by traction during excision.

\section{Post-Operative Care.}

Only one aspect of the subsequent care of the case will be mentioned. There has been a tendency to hasten weight-bearing by allowing the patient up as soon as the fifth day. Most surgeons now consider this too early, and find that post-operative effusions are much less common when weight-bearing is delayed for ten to fourteen days. The longer period allows time for the joint reaction to subside and for a full course of graduated non-weight-bearing exercises.

\section{REFERENCES}

(I) BRISTOW, W. R. (1937), Injuries and Displacements of Semilunar Cartilages," Post-Grad. Med. Jour. 13, 366.

(2) FAIRBANKS, H. A. T. (I942), Non-touch Technique for Simple Fractures, Brit. Med. Jour. (ii), 388.

(3) LAMBRINUDI, C. (1939), Injuries to Both Semilunar Cartilages of the Knee Joint, Proc. Roy. Soc. Med. 32, 635.

(4) BRANTIGAN and VÓSHELL (194I), The Ligaments and Menisci of the Knee-Joint. J. Bone and Joint Surg. $23,59$.

For a short general account and list of easily available references, consult Watson-Jones-Fractures and other Bone and Joint Injuries, Edinburgh, I941. Edn, 2, 544.

\section{Practicalities}

\author{
(Vol. I. No. 7.)

\section{THE TREATMENT OF SOME OF THE CARDIAC DISEASES}

\author{
By F. CROXON DELLER, M.D., D.A.
}

Rest for the patient is one of the oldest forms of treatment of cardiac disease. The cardiac muscle is incapable by itself of rest, and all that one may hope to achieve is a lowering of the patient's metabolic rate, and the removal of all sudden exertions or stresses so that the heart may enjoy a modicum of relaxation. Thus it has been the custom to put a patient who is suffering from severe cardiac failure or an acute affliction of the myocardium to bed on absolute rest, and to keep him there, maybe for months on end, allowing little mental or physical stimulation of any sort. This line of treatment has not been questioned. In the circumstances, is it surprising that most of our cardiac patients become introspective and neurotic in disposition? Is it surprising that when at last they are allowed up they often have multiple symptoms pointing to the heart? Is it surprising that their convalescence is usually prolonged and that a full recovery of function may never be achieved?

How well these questions are answered by a consideration of the symptoms and the mental state of patients who have suffered from a previous rheumatic carditis. Only the other day we saw a young man of twenty-eight, with gross aortic stenosis of rheumatic origin. He refused to consider himself an invalid, playing cricket all the summer, and doing physical exercises during the winter to keep himself fit, as well as doing a full day's work in a war factory. $\mathrm{He}$ was annoyed that he, the eldest of four brothers, should have to remain in civilian clothes, and he failed to understand why this was so, since he felt so fit. Compare this with the other common type of patient, who has a lesser lesion, but who complains of a greater multiplicity of symptoms and is functionally, and actively, incapacitated by the past disease process.

Lately, due directly to the influences of this war, a word new to the medical vocabulary has been brought into prominence. That word is "rehabilitation." In its broadest sense "rehabilitation" means the restoration of the subject to a prior healthy mental state, as well as restoring anatomical continuity wherever possible. We know from our study of pathological anatomy that it may be impossible in the present state of our knowledge to reconstruct the architecture of the cardiac valves once they have been smitten by rheumatic disease. That much must we admit; but, handled in the right way, and retaining a correct perspective of the disease processes, how much may we treat, and with great success, the mental processes of the patient, and, to a lesser degree, the physical powers which that patient can regain when given the right circumstances and thorough understanding. Rehabilitation has not, as yet, been directly 